\title{
How Can Thought Select Between Coincident Material Things?
}

\author{
RORY MADDEN
}

\section{Introduction}

A sculptor finds a hunk of clay in her studio. She creates a statue from the hunk of clay without thereby destroying the hunk of clay. What is the relationship between the statue and the hunk of clay?

The statue has the feature of being brought into existence by the work of the sculptor. The hunk of clay does not have the feature of being brought into existence by the work of the sculptor. The statue has the feature of being well made. The hunk of clay does not have the feature of being well made. The statue has the feature of vulnerability to destruction by flattening of the clay into a large thin sheet. The hunk of clay does not have the feature of vulnerability to destruction by flattening of the clay into a large thin sheet.

It follows from these observations that the statue and the hunk of clay are two things and not one thing. Things that differ in their features cannot be numerically identical.

However, if one is asked to imagine a pair of material things, it is highly unlikely that this is the sort of pair that would spontaneously come to mind. Imagination defaults to prototypical instances. If asked to imagine a fish, one is highly unlikely to imagine a seahorse. One is much more likely to imagine something that looks like a herring. A prototypical pair of material things would be a pair such as two coffee mugs sitting side by side on a table top. They evidently differ in their matter and location. The statue and the hunk of clay are not a prototypical pair of material things. Due to the way that one has been made from the other, the statue and the hunk of clay share their matter and share their exact location. In short, they coincide.

For helpful discussions of the issues I would like to thank Julian Bacharach, Alex Geddes, Charles Jansen, Nick Jones, Mike Martin, and other participants of a research seminar at UCL. 
If asked, without any special priming, to imagine whether a pair of material things could come to be in the same place at the same time, one is likely to begin by imagining a prototypical pair of material things, that is, a non-coinciding pair of material things. Then one will hit a block when one tries to imagine how they might get from their distinct locations into the same place at the same time. Salient acts of pushing and squeezing would destroy one or both things before they could come to be in the same place at the same time. Prototypical pairs of material things physically exclude each other. They cannot coincide.

To the extent, then, that our intuitive responses depend on our imagination and our imagination defaults to prototypes, there will always be something strange or unintuitive sounding about the view that distinct material things can coincide. But of course, this is not serious evidence against the view, any more than our tendency to imagine prototypical fish is serious evidence against the view that seahorses are fish. Call the thesis that distinct material things may coincide at a time pluralism. Call the thesis that distinct material things may not coincide at a time monism. ${ }^{1}$ Are there more serious reasons to dislike pluralism and to favour monism?

A potentially more serious difficulty for pluralism goes by the name of the 'grounding problem'. The grounding problem, in general, is the difficulty of explaining, or grounding, the putative differences between coincident material things, given that they have so much underlying in common. ${ }^{2}$ For example, a standardly posed aspect of the problem is this: given that the statue and the hunk of clay share the same underlying microphysical parts, intrinsically configured in the same way and situated in the very same environment, how could one thing, but not the other, have the power to resist destruction by flattening? In virtue of what could two things, otherwise so similar, differ in this broadly modal respect?

In order to develop this challenge into a serious argument, the opponent of pluralism is likely to need to rely on a strong metaphysical principle about the supervenience of a thing's macroscopic level properties upon the properties of its very small parts. While I cannot defend the point here, I believe the pluralist can reasonably deny such a principle.

My aim in this essay is to explore an aspect of the grounding problem for pluralism that does not rely on such a principle. The difference that

1 I borrow the terminology of 'pluralism' and 'monism' from Fine 2003.

2 The name 'grounding problem' is from Bennett 2004. Earlier presentations of the problem can be found in Sosa 1987 and Burke 1992. 
calls for explanation is this. Suppose that one goes to a sculpture gallery. Looking at a certain statue one comes to think that it is nicely made. According to the pluralist, however, there is a second material thing, the hunk of clay, which also lies in the direction of one's gaze. How is it, then, that my thought comes to focus on the one but not the other material thing? What grounds the pairwise difference with respect to the relational property of being the object of my thought, given the otherwise extensive similarity of the pair? Let us call the problem of explaining how a subject may think of one of a pair of coincident material things the selection problem.

I begin in the next section by reviewing a traditional sortalist approach to the selection problem. I will discuss some standard counterexamples to sortalism and criticise some broadly sortalist replies to these counterexamples. The shortcomings of sortalism have motivated a number of writers recently to propose what I will call in the following section a perceptualist alternative, according to which perception alone can suffice to resolve the object of thought in cases of material coincidence. I criticise this approach and draw out some consequences about visual experience. In the final main section of the essay, I propose an epistemic solution to the selection problem, distinct from both sortalist and perceptualist approaches.

\section{Sortalism}

Sortalism is the view that a thinker can think about an individual object only if the thinker correctly classifies the object according to its sort. An object's sort is a universal feature, typically picked out by a common noun such as 'horse' or 'set'. In contrast with a qualitative feature such as redness, a sort is held to be a feature that determines a principle for counting objects with that feature. A sort determines what Frege (1884: $\$ 62)$ called a 'criterion of identity'.

While the view that determinate reference to an object requires a grasp of its criterion of identity has its roots in Frege's discussion of numbers and other abstract objects, sortalism has also been asserted in various distinguished twentieth century discussions of the possibility of reference to material things (Quine 1950; Strawson 1959, ch. 6; Dummett 1973, ch. 16; Wiggins 2001). Indeed, sortalism has frequently been motivated as a solution to something very like our selection problem. Here is Quine seeking an explanation of how one can 
refer to a river, rather than, say, the particular portion of water that constitutes the part of the river in one's immediate vicinity:

Pointing is of itself ambiguous as to the temporal spread of the indicated object. Moreover, even given that the indicated object is to be a process with considerable temporal spread, and hence a summation of momentary objects, still pointing does not tell us which summation of momentary objects is intended, beyond the fact that the momentary object at hand is to be in the desired summation. Pointing to $a$, if construed as referring to a time-extended process and not merely to the momentary object $a$, could be interpreted either as referring to the River Caÿster of which $a$ and $b$ are stages, or as referring to the water of which $a$ and $c$ are stages, or as referring to any one of an unlimited number of further less natural summations to which $a$ also belongs [...] Such ambiguity is commonly resolved by accompanying the pointing with such words as 'this river', thus appealing to a prior concept of a river as one distinctive type of time-consuming process, one distinctive form of summation of momentary objects. (Quine 1950: 622-3) ${ }^{3}$

More recently, Wiggins raises a selection problem for the view that merely pointing towards an object in a location could be enough to select an object of thought:

There will exist too indefinitely many items with too many distinct principles of identity and persistence which you might find in that place - the thing, the parcel of stuff that makes up the thing, and the mereological sum of all the components of that parcel, to name but three. (Wiggins 2001:150 n. 13) ${ }^{4}$

Wiggins's solution to the problem constitutes the psychological, or cognitive, component of his complex views about the 'sortal dependency of individuation':

3 Notice that Quine's claim in this passage is only that appeal to a prior concept of a sort is a common way to resolve the ambiguity. He is not claiming that it is the only way. An alternative, he goes on to suggest, is that a thinker without a prior concept may eventually resolve the ambiguity by cottoning on inductively to a criterion of identity that strikes him as naturally unifying a series of things ostended for him (Quine 1950: 623). But even if a prior concept is not required, for Quine it remains the case that determinate reference awaits the thinker's grasp of a correct criterion of identity.

4 I take it that a parcel of matter, of which our hunk of clay is an example, is distinguished from the mereological sum of its components by some requirement of integrity. A mereological sum, being defined solely in terms of its parts, need not be integrated, but may exist entirely scattered. I ignore mereological sums henceforth in this essay, focusing only on kinds that figure in ordinary thought, such as animals, artefacts, and hunks of matter. 
For a thinker to single out or individuate a substance, there needs to be something about what he does, something about his rapport with $x$ or his relational state towards $x$ and his practical sensibility in relation to $x$, which ... sufficiently approximates to this: the thinker's singling $x$ out as $x$ and as a thing of a kind $\mathrm{f}$ such that membership in $\mathrm{f}$ entails some correct answer to the question 'what is $x$ ?' (Wiggins 2001: 7)

Although there are subtleties in Wiggins's statement, we can again see the idea that singling out a thing requires more on the part of the thinker than merely pointing or looking at a location. In addition, the thinker must, in some way, grasp the kind or sort of the thing in question.

Wiggins's statement also presents a useful opportunity to clarify the sortalist claim that a thinker can think about an individual object only if the thinker correctly classifies the object according to its sort.

Suppose that I formulate the following definite description: 'the first object Donald thought about when he awoke yesterday morning'. Was it his wife, his new golf club, a prize-winning trout? I have no idea. Suppose it was his new golf club. Then in some sense the golf club is in fact the object of my description. It is the thing that fits the description that I have framed. But I have not myself classified it according to the sort of thing it is. I have no opinion about its sort. Is this a straightforward counterexample to sortalism?

This problem draws attention to Wiggins's emphasis on requirements for a thinker to 'single out' or 'individuate'. Wiggins explains the achievement he has in mind:

To single $x$ out is to isolate $x$ in experience; to determine or fix upon $x$ in particular by drawing its spatio-temporal boundaries and distinguishing it in its environment from other things of like and unlike kinds (at this, that and the other times during its life history); hence to articulate or segment reality in such a way as to discover $x$ there [...] And one may well refer to $x$, of course, without in our primary sense singling out $x$ at all. (Wiggins 2001: 6)

So, the condition that one singles $x$ out is relatively strong. One could, as above, frame a description (which is 'about' $x$ insofar as its truth or falsity in fact turns on the condition of $x$ ) without thereby in any intuitive sense isolating $x$ in experience. In what follows, I shall understand the notion of 'thinking about an object' as it features in sortalism relatively narrowly, as an achievement involving Wigginsian singling out. I won't try to make Wiggins's metaphors more precise, but 
a paradigm of thinking about an object in this sense will be a perceptual demonstrative thought, an episode of thinking directed by perception onto an object present, prototypically expressed by a demonstrative pronoun 'this'.

Even understood more narrowly, the sortalist claim seems vulnerable to counterexamples. It seems quite possible to single out and thereby think about an object that one has not correctly classified according to its sort. ${ }^{5}$

Suppose that one is given a mystery object to look after: one is unsure whether it is a large tightly curled-up crustacean, a sculpture or a fossil. One puts it on the mantelpiece. It seems obvious that one can single it out and think about it as 'this' while remaining entirely neutral about its sort.

One can also make mistakes about the sort of object one is singling out. Suppose in the sculpture gallery one mistakes a dozing security guard for a statue. Again, it seems intuitive that one could single him out and think about him while failing correctly to classify him according to his sort.

How can the sortalist respond to such cases? Setting aside the unappealing option of denying that the thinker really singles out an object, the remaining options must develop in one way or another a suitably permissive understanding of 'correctly classifies the object according to its sort'. I will consider three such options.

Wiggins's paradigmatic sorts are Aristotelian secondary substances such as horse or man. But one response is to understand sortalism to require correct classification at only some higher or more abstract level. Lowe, for example, has defended a variant of sortalism that he calls categorialism. ${ }^{6}$ According to categorialism, 'a thinker can single out an object only if he or she grasps, at least implicitly, some categorial concept under which he or she conceives the object in question to fall ... such categorial concepts would be the concept of an animal, a material artefact, and ... a geographical prominence' (Lowe 2013: 11).

Lowe's categorialism, while somewhat permissive, does not fare any better with the two problem cases given. The neutrality and error in these cases are transcategorial. In the first case, one is neutral between

5 For the following style of counterexample to sortalism see Ayers 1974; Hirsch 1982, ch. 3; Campbell 2002, ch. 4; Snowdon 2009.

6 Lowe 2007; 2013, ch. 2. 
animal and material artefact. In the second case, one mistakes an animal for a material artefact. In neither case does the thinker correctly conceptualise the thing according its broad category. ${ }^{7}$

In the face of this, the sortalist might try ascending even higher up the hierarchy of subsuming classifications, to a transcategorial level, which includes, say, material thing. For it will be noted that in each of the problem cases one still correctly takes the object of thought to be a material thing, even if one does not get its specific category right.

There are two difficulties with this move. First, this highly permissive requirement for correct classification appears still to face counterexamples. Suppose that one mistakenly takes for a statue what is in fact a clever hologram among a group of statues. It seems obvious that one could still single out the hologram and think the (false) thought 'that is a statue', despite failing to classify it correctly at even this high level. The correct high-level classification would not be material thing but perhaps something like purely optical object. A converse error is also possible: one could take for a hologram what is in fact a glass statue. Finally, one could be simply neutral as to whether it is a material thing or not and refrain from classifying it either way.

The second difficulty is more local to present concerns. As soon as sortalism is framed at the level of material thing or higher, it becomes difficult to see how sortalism can solve the selection problem for pluralism. In the basic case used initially to pose the selection problem, both the statue and the hunk of clay are material things. In one version of the case, the thinker classifies the object of thought correctly as a statue, so sortal classification might in principle explain selection. But suppose the case is varied so that the thinker wrongly takes the statue for a human being. It remains, it must be said, strongly intuitive that the thinker, in thinking 'that is a human being', would still be singling out the statue rather than the hunk of clay and mistaking the statue for a human being. The thinker is correct in taking the statue to be a material thing, but the problem is that this correct high-level classification on the thinker's part does not discriminate between the statue and the hunk of clay. The hunk of clay is also a material thing. Hence it cannot be explaining the selectivity of thought in this case.

7 Lowe swallows the implausible consequence that in such cases one fails to think about the object in question. 
The second and third sortalist replies to cases of neutrality and error are similarly idle when it comes to explaining the selectivity of thought.

The second reply turns on the plausible point that thought and knowledge are sensitive to mode of presentation. I can know that this man is wearing a hat without knowing that Smith is wearing a hat, even if this man is Smith, for I could fail to know that this man is Smith. In this case a particular individual is presented in more than one way. Universal sorts or kinds can also be presented in more than one way. Talk of correctly 'classifying' an object according to its kind suggests that the object's kind is presented to the thinker in a contextindependent way that somehow places it in a larger system of contextindependently presented kinds, if not via Linnaean binomials like Homo sapiens or Equus ferus then simply via 'human being' or 'horse'. But one could instead refer to a sort in a context-dependent, demonstrative manner as this sort. This is the possibility exploited in a Putnamian deictic account of reference-fixing for natural kind terms in the primal situation of ignorance of the kind's real nature, for example in fixing the reference of 'gold', in a situation of ignorance of its atomic structure, as simply this stuff. ${ }^{8}$

This point yields the following sortalist response to the problem cases of neutrality and error. Although the thinker fails to think the correct classificatory thought ('this is a statue', say), the thinker could still correctly classify the object as an object of this sort; the thinker could still think the correct classificatory thought 'this thing belongs to this sort'. So the cases are no longer clear counterexamples to the sortalist thesis that a thinker must correctly classify the object of thought according to its kind. ${ }^{9}$

Even if this move saves the letter of sortalism, the selection problem remains intractable. The difficulty is that ostension of kinds and other universal features appears to be dependent upon ostension of instances of the feature in question. For example, the salient way to pick out that colour is by ostending an individual that instantiates the colour. Now, take the case of the thinker who mistakes a statue for a human being. Given that the thinker can single out the individual statue, the thinker is

8 Wiggins emphasises that our conception of a natural kind can be deictic (2001: 77-90).

9 Dickie (2011: 53-4) appeals to ostensive knowledge of kind in reply to similar counterexamples on behalf a version of sortalism. In more recent work, she appears to have abandoned sortalism (2015: 131-6). 
thereby put in a position to demonstrate its universal kind and to correctly classify it as a thing of this sort. But the problem was to explain how the thinker can single out the individual statue in the first place. The possibility of ostension of kind seems not to explain this, but on the contrary to be explained by the thinker's singling out of the individual which instantiates the kind.

Would it help the sortalist to insist that the possibilities of demonstrative reference to sort and demonstrative reference to individual object stand in a relation of 'mutual dependence' (Wiggins 1980: 219)? It is difficult to see how this could help. Stay with the subject who singles out a statue as this while mistaking it for a human being. Suppose, as Wiggins suggests, that thought of the statue as this (individual) and the thought of its sort as this (sort) arise coevally, as mutually dependent parts of a single package. The question remains: how is it that these two mutually dependent demonstrative thoughts arise together, rather than two mutually dependent demonstrative thoughts referring respectively to the individual hunk of clay and to its general sort bunk of matter? The selectivity of thought remains unexplained.

The third reply is suggested by Wiggins's remark that in cases of ignorance, where one lacks any 'in the context informative' answer to the question 'what is it?', the presence of the thing would still give the thinker 'assurance that $(\exists \mathrm{f})$ ( $\mathrm{f}$ is a well-defined thing kind, and this strange thing belongs in f)' (Wiggins 1980: 218). In other words, even if there is no kind such that one correctly thinks that the thing is of that kind, one may still be correct in thinking the existential thought that there is some kind such that the thing is of the kind.

Suppose that sortalism is weakened to require as a condition of singling out an object of thought only this minimal general correctness about sort. Then sortalism may well be true. But this move, perhaps even more obviously than the appeal to ostensive knowledge of kind, leaves it mysterious how sortalism could explain how the thinker's thought selects between the statue and the hunk of clay in a case of error or neutrality. The two material things are after all both things of some kind.

\section{Perceptualism}

Our counterexamples to sortalism, in which it seems intuitive that a thinker is singling out an object despite failing to classify it correctly, 
are notably examples in which the subject sees the object in question. Counterexamples to sortalism involving thoughts about imperceptible abstract objects, like sets or numbers, do not so readily come to mind and, as we shall see in the following section, sortalism may be closer to the truth when the thesis is restricted to such things. So a natural proposal, one in fact popular among recent opponents of sortalism, is to claim that perceptual experience can suffice to resolve ambiguity of thought about ordinary concrete things in our environment, without the need for supplementation by correct sortal classification.

Recent developments in vision science have encouraged some writers to this position. The Quinean problematic seems to presuppose that pointing or attending unsupplemented at best serves to focus upon some matter-filled, or quality-filled, region in the thinker's line of sight. If so, then in order to single out determinately just one of the multiple objects passing through that region, one must appeal to a further mechanism of conceptual selection. But in fact, 'locationbased' theories of visual processing have made room in recent years for 'object-based' theories. The consensus now seems to be that preconceptual visual processing can deliver experience of a scene already segmented into 'visual objects', where a visual object is some common bearer of a range of visible properties, tracked through changes in its location and other properties, with a speed and efficiency characteristic of low-level or pre-attentive processes. ${ }^{10}$

This has suggested to writers such as Campbell that 'the work that [sortalism] allots to grasp of sortal concepts ought rather to be assigned to the principles used by the visual system in binding together the various characteristics of a single object' (Campbell 2002: 71). Dickie is an ally in this cause:

The empirical evidence ... undermines ... the claim that conceptually unaided mental or physical pointing is ambiguous. For this evidence suggests that our pre-conceptual processing parses the visual field into visual objects. So it suggests that the boundaries that proponents of the traditional argument think sortal concepts draw are already there in the pre-conceptual deliverances of our perceptual systems. (Dickie 2011: 41) ${ }^{11}$

10 See Pylyshyn 2003, ch. 4 for a review of evidence.

11 Similar claims are made on behalf of perception by Rachel Goodman:

'My suggestion is that the sortalist is wrong to think that picking out a particular object for thought requires employing a property concept (in particular, a sortal concept) as part of one's act of individuation. Rather, we should recognize that, 
If these writers are correct about the empirical evidence, then the evidence only confirms what for other writers is simply the result of commonsense reflection on our experience of the world. Ayers, for example, takes it to be obvious that 'typical material objects or things are the naturally discrete and unitary objects presented in animal experience' (Ayers 2005: 535). An ordinary object 'does not wait to be sliced out of reality by some human concept. It is presented to us as the unitary and discrete individual that it is' (Ayers 1991: 194). Snowdon criticises the sortalist solution to the selection problem for overlooking 'the possibility, to my mind quite plausible [...] that our perceptual experiences have a character that directs our object-oriented thought onto one rather than another object present' (Snowdon 2009: 268).

I want to argue that this perceptualist approach to the selection problem is not in fact promising. I'll begin with a clarification about what perceptualism needs to achieve. It should be agreed on all sides that ordinary sorts such as $d o g$ or statue can be recognised on the basis of vision. Furthermore, sortal categorisation is sometimes reasonably regarded as 'perceptual', for more than one reason. First, as the familiar duck-rabbit demonstration highlights, such categorisation can, at least in some sense, make an introspectable difference to the phenomenology of the subject of a visual experience. Second, categorisation of stimuli as falling under ordinary sorts can be achieved on the basis of remarkably brief stimuli, presentations shorter than the shortest recorded blink of the eye. It is arguable that this speed of processing simply does not leave time for the intervention of top-down influence from central cognition. The sortal categorisation here must be the output of a relatively early stage or module in visual processing. ${ }^{12}$

However, recognising that perception itself can involve sortal categorisation does not really constitute any progress with the selection problem. To our case of singling out in sortal error, we can make the following addition. As well as mistakenly ascribing the sort human being in their thought or judgement, the subject also rapidly perceptually categorises something as human being. It is still plausible that the

the application of principles of individuation comes "built in" to our perceptual abilities' (Goodman 2012: 94).

12 Mandelbaum (2017) makes this argument. He does not deny that there can be a prior learning period in which central cognition does act upon this module over time. The categories in question need not be innate. 
subject can single out the statue and not the hunk of clay as 'this'. If perception is somehow explaining the selectivity of thought here, then it is not because perceptual experience itself is correctly categorising the object of thought.

So how then could perception solve the selection problem? It would have to be the case that perception can somehow present, for attention and demonstrative singling out as 'this', some objects, but not others, in the field of view. There is plenty of evidence that relatively early visual processing can in some ways do just this. Studies of automatic spread of attention, ${ }^{13}$ amodal completion ${ }^{14}$ and multiple objecttracking ${ }^{15}$ demonstrate that certain objects can be privileged as potential objects of attention and singling out. The question is: which objects? This is a matter of ongoing research, but the evidence is that the visual system tends to select whole three-dimensional objects, rather than just the visible portions of their surfaces. More specifically these are things with cohesiveness, boundaries and continuous spatiotemporal trajectories. ${ }^{16}$

It follows that vision alone can forestall some of the ambiguity that sortal concepts have traditionally been invoked to resolve. In a typical situation, a demonstrative used in response to visually presented things will not pick out a mere pattern of light, a visible part of a surface, let alone some gerrymandered fusion of arbitrary objects. One's attention will have been drawn by some whole object.

But what does not follow from this, nor from any study of which I am aware, is that vision would select between the statue and the hunk of clay. Both of these objects are cohesive, bounded objects with continuous spatio-temporal trajectories. Both objects meet the ordinary conditions for selection by the visual system. Indeed, they exactly share their place, shape, orientation, motion, colour, texture and every other cue available to vision. Of course, the hunk of clay, but the not the statue, could persist extruded into a filament too fine to see or flattened into a huge flat shape whose boundaries outrun the thinker's field of view. The problem is that when coincident with the statue, the hunk of

13 Duncan 1984; Scholl 2002. ${ }^{14}$ Driver et al. 2002. ${ }^{15}$ Scholl et al. 2001.

16 See Burge 2010: 437-71 for a wide-ranging review of the evidence that individual cohesive, bounded 'bodies' in this sense are primary objects of vision, and not just objects for conceptual thought. See Scholl et al. 2001 for a study of the role of properties such as physical connectedness in attracting object-based attention. 
clay meets the conditions for visual selection, and it does so precisely as well as the statue.

Dickie makes a brief attempt to support the idea that vision selects one rather than another of a pair of coincident material things:

[It] is if, but only if, you are attending to the piece of metal of which the chair is made, rather than to the chair itself, that your attentional link will remain intact through a change that the piece of metal but not the chair survives. (Dickie 2011: 41) ${ }^{17}$

This may be right. But it is hardly evidence that visual processing is attuned to only the piece of metal before the chair is destroyed. The alternative, and more natural reading, is that the thinker in this situation is interested in the piece of metal, which is recognisably still present, so they keep on looking at it. It is a perfectly ordinary case of pre-perceptual orientation and maintenance of attention by a subject's practical interests in a certain kind of thing. It is not evidence that the visual system was only ever locked on to the piece of metal.

Here is an analogy to reinforce the point. Suppose that one is very interested in blackbirds and not at all interested in thrushes. One sees from a great distance what one takes to be a single blackbird but which is in fact a blackbird and a thrush very closely circling each other. The pair then come close enough to be told apart by vision and the thrush flies off. One's eyes stays firmly on the blackbird. Given your sustained overriding interest in blackbirds, there is perhaps some slight plausibility to the suggestion that your thought 'this' was focused on the blackbird all along. But what does not seem at all plausible is that your maintaining firm attention on the blackbird after they go their separate ways in any way supports the view that your visual system was uniquely locked on to the blackbird before they went their separate ways. The two birds were just visually indiscriminable at that stage.

So, as it seems to me, the perfect visual indistinguishability of the statue and the hunk of clay make a perceptualist solution to the

17 Campbell also offers only brief comments along the same lines as Dickie (Campbell 2002: 70, 75). Goodman (2012) suggests that our perceptual systems resolve statue-clay ambiguity and part-whole ambiguity but then, like Dickie, presents evidence relevant only to the resolution of part-whole ambiguity.

The explanation for the sketchy character of these writers' comments about the statue-clay selection problem may simply be that these writers are in fact inclined to the monist position, according to which there is no problem here to be solved. 
pluralist's selection problem extremely unlikely. The pluralist should look elsewhere.

But before leaving perception behind, the pluralist ought to face up to the question: what does the subject see in a case of material coincidence?

When faced with a statue in normal viewing conditions, one would pre-theoretically take oneself to be seeing a single thing (and its parts). That's how one might innocently describe one's situation. Can the pluralist respect this appearance? The difficulty just noted is that the two material things are equals as far as the visual system is concerned; it is hard to justify the claim that vision presents just the statue or just the hunk of clay.

One way then for the pluralist to validate the appearance that there is a single object seen is by finding some third object, distinct from either the statue and the hunk of clay, to be the single thing seen. How might this go? The way in which vision scientists write of 'visual objects' sometimes suggests that these segmentations of the visual field are not to be identified with ordinary material things in the distal environment. They are in some sense subjective things, essentially related to vision. ${ }^{18}$ For example, in the case just considered of the two distant birds, one might identify the object of vision with a single $d o t$, a visual object distinct from either bird. Likewise, it might be suggested, in the case of the indistinguishable statue and the clay, one could claim that one indeed sees a single thing, but what one sees is a visual object.

I don't think that this is the way to go. First, it is completely unclear what a visual object in this metaphysical sense is supposed to be. A more intelligible notion of visual object would parallel the notion of an object of thought. The visual objects are not a special metaphysical category of subjective objects but simply whichever things vision selects, just as an object of thought is anything thought about. These could be things of quite different sorts: rainbows, holograms, shadows, as well as material things. Second, if the cost of securing the intuitive claim that one sees only one object is that underlying ordinary things like statues and human beings are strictly speaking invisible in perfectly normal viewing conditions, then perhaps the pluralist would be better off giving up the claim that one sees only one thing.

18 For examples of such suggestions about visual objects, see Wolfe and Bennett 1997 and Pylyshyn 2000, 2001. 
So, the following is the optimal pluralist claim about vision in the statue-clay case. It is not the case that one sees no material thing, and it is not the case that one sees only one material thing. One sees two material things. This is counterintuitive; it is not how a viewer would describe their situation pre-theoretically. But the viewer's impression that there is only one object to be seen receives a ready explanation from the pluralist. The two objects are entirely indistinguishable by vision.

A corresponding pre-theoretical appearance concerns not just our visual situation but our conceptual situation. When faced with a statue in normal conditions, it does not only seem to one as if one is seeing a single object; it also seems to one as if one's visual experience alone enables one to select an object in thought, to single it out as 'this'. There seems to be a purely experiential basis for selection of object of thought. The pluralist must now concede that this appearance too is mistaken. Given the double visual presentation of objects in the case of ordinary material things, perhaps the only clear examples of objects genuinely selected for thought on the basis of visual selection alone will be purely optical objects, like rainbows or holograms, that do not coincide with hunks of matter.

The puzzle then is to explain what else does enable singling out of ordinary material objects in simple demonstrative thought. One has no introspectable sense of engaging in any further act of selection over and above simply looking at a thing. But without uncovering a decent explanation, the pluralist will find it hard to sideline convincingly the more obvious monist explanation of why things seem this way: that vision seems to be enough to enable one to select an object of demonstrative thought because it is enough; there is really only one material thing there to see and think about.

\section{Knowledge and Selection}

Gareth Evans's position in Varieties of Reference is pluralist: 'a $G$ may be able to share a position with a thing of a different kind: for instance a statue and a piece of clay' (Evans 1982: 107). But his position is also anti-sortalist: he observes that it does not appear to be true that demonstrative identification must be accompanied by a sortal which sets the boundaries of the thing in space and time' $(1982: 178) .{ }^{19}$ So,

19 It should be noted that Evans does hold that possession of something called a 'fundamental Idea' of a material thing requires grasp of its sort (1982: 107). 
one might wonder, what if anything does he have to say about the selection problem? He makes the following interesting, though undeveloped, remarks:

The idea of discovering the sort of a thing, identified demonstratively, would not make sense if there was not some ranking of sorts. As Trinculo goes along the beach and espies Caliban for the first time, he asks 'What is this?' It must be presumed that 'This is a living animal' is (at least) a better answer than 'This is a collection of molecules'. Similarly, when the fisherman wonders what he has at the end of his line, the answer 'A statue' is a better answer than 'A piece of clay'. Since we seem to know this ranking, it is not important for us to enquire into its principles: a determinate answer can be given to the question 'What kind of thing is this?' provided a definitely extended object is indicated and such an indication does not by itself presuppose any sortal. (Evans 1982: 178-9)

Let material constitutee be the transcategorial sort subsuming the categories animal, plant, functional artefact, material work of art... but not hunk of matter. Material constitutees are the ordinary things that, according to the pluralist, coincide with mere hunks of matter. Evans's suggestion, plausible as far as it goes, is that material constitutees in some sense 'outrank' hunks of matter as default objects of demonstrative identification in cases of sortal ignorance. But what could the 'principles' of this ranking be? What could explain the statue's dominance over the hunk of clay in this ranking?

A vague but true starting point for enquiring into the principles of this ranking is the observation that material constitutees are typically more interesting than hunks of matter. They are more interesting in more than one respect. Material constitutees are typically more interesting in the respect that there is literally more to learn about them than there is to learn about hunks of matter. While the statue and the hunk of clay share many properties - shape, colour, solidity, matter - the statue has in addition a further broad range of qualities appropriate to its sort, such as its value, artistic genre and style. The hunk of clay has no similarly broad range of further qualities proprietary to its sort. There is plausibly a similar asymmetry between the sorts human being

A fundamental Idea of a thing is a way of thinking of the thing in terms of the features which in fact metaphysically ground its distinctness from all other things (1982: $\$ 4.4)$. Evans's (correct) view is that a demonstrative identification need not be a fundamental Idea in this sense. 
and body. Both are warm fleshy objects of a certain spatial form. But the human being has, in addition, a further range of biological and psychological features: the human being, but not its body, eats, socialises, sleeps, perceives and thinks. ${ }^{20}$

Even if one is sceptical about the quantitative claim that material constitutees have more character than hunks of matter, it can hardly be doubted that the particular character of material constitutees makes them of far greater interest to us than hunks of matter. Our practical interests are largely focused upon such things - above all, our fellow human beings, but also our various pets, plants, tools, gadgets and furniture. While the visual system is promiscuous, attracted to all sorts of bounded, cohesive material wholes and anything sufficiently visually similar to such wholes, our human practical interests are more particular.

I believe the task for the pluralist who wishes to solve the selection problem is to develop an account of thought content determination that somehow engages with these observations about our predominant interest in material constitutees.

A simple idea would be to develop a permissive variant of sortalism according to which thought about an object is focused by correct classification at the relatively high-level material constitutee. This level is higher than Lowe's categories of animal or artefact, but lower and more specific than the undiscriminating material thing. This variant promises to explain how even in a case of neutrality or error about whether the thing is, say, statue or human, one is nevertheless classifying it correctly at a level that distinguishes it from the hunk of matter.

The problem for this simple idea has already been raised: singling out seems compatible with mistakes or ignorance, even at such a high level. One can be neutral or mistaken about whether the thing that one is singling out in thought is a material constitutee: it could be a hologram, a shadow, an image moving on a screen.

20 Burke (1994: 610-14) makes the point that sortal concepts can differ in the breadth of the range of properties entailed by their satisfaction. He makes the point in the course of developing his 'dominant sortals' version of monism, on which the statue dominates out of existence the pre-existing hunk of clay. My interest is in explaining what might be called the mere 'cognitive' dominance of the sort statue. See Fine 2003: 206-8 for more examples of properties proprietary to various sorts of what I am calling material constitutee. 
In what follows, I shall defend a subtler theoretical vindication of Evans's 'ranking' by appealing to a principle of charity in the determination of thought content. According to such a principle, the right assignment of content to the thoughts of an individual or community is the assignment that best makes the thoughts overall truthful, errorfree, knowledgeable, rational, intelligible or in some other way virtuous. $^{21}$ This is not the place for a serious critical comparison of different charitable approaches to content determination. For present purposes, I am simply going to assume a certain plausible theory, to illustrate how the pluralist might theoretically account for determinate thought content while committing to neither sortalism nor perceptualism. The particular charitable theory I shall assume is an epistemic theory, recently defended by Williamson. According to this theory, the right assignment of thought content is the assignment that maximises knowledge. ${ }^{22}$ This theory has the advantage over a simple truthmaximising theory that it rules out various 'crazy' assignments that ascribe to a thinker accidentally true thoughts about things that all lie beyond the awareness of the thinker.

An immediately appealing feature of the epistemic theory is this. It is not entailed that a thinker must have any particular piece of knowledge about the object of thought. Error or ignorance in any particular

21 See Davidson 1984 and Lewis 1974 for classic statements of charity-based theories of content.

22 'Given the central role of knowledge in intelligent life, the intimate relation between knowledge and reference is hardly surprising. Reference maximizes knowledge because its role is to serve knowledge, not to impose any independent limitation on it' (Williamson 2007: 270). Other authors have defended closely related ideas. Evans's well-known proposal that the reference of a proper name is the 'dominant source of information' is closely related to a knowledgemaximising principle: Evans defines information in terms of causal relations 'apt for producing knowledge' (Evans 1973: 15). The theme is elaborated by McDowell in a discussion of interpretation: 'One cannot intelligibly regard a person as having a belief about a particular concrete object if one cannot see him as having been exposed to the causal influence of the object in ways suitable for the acquisition of information (or misinformation) about it' (McDowell 1977: 136). Brewer argues that the 'process of making sense of what people are talking about is constrained precisely by considerations of what they have knowledge about' (Brewer 2000: 426). A more recent comparison is Recanati's (2012) view that the reference of 'mental files' is fixed what he calls 'epistemically rewarding relations', in effect ways of coming to know about the referents. Dickie's recent theory (2015) that the object of a body of beliefs is the object that renders the beliefs 'justified' and 'non-luckily true' studiously avoids appeal to knowledge but will obviously deliver similar results. 
respect - even serious classificatory error or ignorance - can in principle be compensated by the attribution of other knowledge about the object, so long as knowledge is maximised overall. To illustrate this feature, stay with the case where the subject thinks about a visible hologram as 'this' while wrongly taking it to be a material statue. Although the thinker lacks classificatory knowledge of the thing, the thinker does still have a rich channel of knowledge of the thing: the thinker is in a position to know a range of its visible properties, such as its location and colour. Assignment of the hologram to 'this' is therefore the assignment that maximises the subject's knowledge in this situation of sortal ignorance. This assigns plenty of visually based knowledge, and there is no better candidate.

The epistemic approach also predicts the difficulty in finding intuitive cases of a thinker singling out an abstract object in a situation of sortal ignorance, and hence why sortalism has more plausibility when restricted to thought about such objects. How? The ways in which one can come to know various truths about a set or a number typically involve operations or inferences resting essentially on the premise that the thing in question is indeed a set or a number. So, typically, if one doesn't know that the thing is a set or a number, then one won't be in a position to know anything about it. Correspondingly, it is implausible to suppose that one's thought might have selected it. In contrast, one can be linked to things in space and time by perceptual channels of knowledge that do not rest upon knowledge of sort. ${ }^{23}$

Let us return now to the troublesome case of coinciding material things in a case of sortal ignorance. Visual knowledge channels alone could ground thought about the hologram in sortal ignorance, because in that case there was no second competitor candidate sharing the visual appearance of the hologram. There was a single epistemic best

23 Perhaps there is room to claim that testimony is a potential back-up channel for knowledge of abstracta in a situation of sortal ignorance. Suppose a hearer is told by a speaker, 'it has 256 members'. The speaker is talking knowledgeably about a set but the hearer wrongly believes on the basis of some confusion that the thing being talked about is a learned society with 256 members. There is no such society. The hearer takes themselves to agree to what the speaker said. But would the assignment of the set as object of the thinker's subsequent use of 'it has 256 members' ascribe to the thinker knowledge of the set in this situation of sortal confusion? I wouldn't want to insist that it doesn't, but it is certainly not clear that it does. It is correspondingly unclear, as the epistemic approach predicts, whether the thinker really has anything in mind as object of thought. 
candidate. The troublesome difference is that the statue and the hunk of clay are tied as potential objects of purely visually based knowledge. And since the thinker is mistaken about sort, taking the statue to be a motionless human being, the thinker also fails to have beliefs ascribing sort-proprietary qualities, such as artistic genre, which might otherwise promise to make the statue the candidate that maximises the thinker's knowledge.

Even if we do not make correct high-level classification a universally necessary condition of thought, could we not in this case appeal to the thinker's knowledge of the statue that it is a material constitutee? This strategy is difficult to execute. First, we can reasonably stipulate that thinker does not possess the abstract theoretical concept material constitutee, without diminishing the plausibility of the view that the thinker's use of 'that' defaults to the statue not the hunk of clay. But let us suppose that the thinker does, perhaps tacitly, think a thought ascribing the category material constitutee. Does the thinker know of the statue that it is a material constitutee? In the case of sortal neutrality, where the thinker looks at the mystery object on the mantelpiece, it is possible that the thinker with the concept could know that is a material constitutee on the basis of knowing that it is either an animal, or an artefact, or ... But in our case of sortal error, the question of how the thinker is supposed to know of the statue that it is a material constitutee is more problematic. On assignment of the statue to the thinker's use of 'that', the thought 'that is a material constitutee' will rest upon the false lemma that 'that is a human being'. It is commonly thought that knowledge cannot be derived from a false premise in this way.

Similar problems beset versions of the strategy that try to appeal to the thinker's knowledge, not of the sort material constitutee as such, but instead of a modal or dispositional property that distinguishes material constitutees of different sorts from hunks of matter, such as would be destroyed by flattening. First, we could stipulate that the thinker does not in fact engage in any such counterfactual speculations. But even if the thinker does, the thinker's basis for ascribing the property to the statue would again be the false premise that it is a human being. If that falsehood is the subject's sole basis for the belief that it would be destroyed by flattening, then it is not very plausible that the belief amounts to knowledge.

One might respond to these problems by questioning the operative 'no false lemmas' requirement for knowledge. Newton's law of 
gravitation is false but, arguably, astronomers were able to come to know facts on its basis, such as the approximate times of solar eclipses. The general point is that, under the right circumstances, a falsehood can still be a reliable or safe basis for forming certain beliefs. One might apply the point here. Although the belief 'this is a material constitutee' is based on the false belief that 'this is a human being', the assignment of the statue to 'this' would still ascribe a quite safely true belief 'this is a material constitutee'. The subject could not easily have been mistaken about that. So perhaps the thinker does have knowledge that discriminates between the statue and the hunk of clay.

Unfortunately for this response, the capacity of the thinker to single out the statue seems robust under variations of the case that would make such putatively discriminating beliefs unsafe. Suppose that the statue which the thinker mistakes for a human being is in a crowd of very similar looking humanoid holograms. Although assignment of the statue to 'this' makes the belief 'this is a material constitutee' true, the basis for the subject's belief - taking at face value a human-like appearance - could in these circumstances very easily have led the subject instead to form a false belief expressible by 'this is a material constitutee'. In the presence of all these nearby immaterial dead ringers, the subject doesn't know of any object present that it is a material constitutee. But the thinker can still single out the statue in thought.

How then to proceed? The pluralist might reply that these problems in explaining the determinacy of thought should be blamed upon the epistemic approach to content determination rather than the view that material things can coincide. It might be thought that we just should look for a different approach to content determination. ${ }^{24}$

This would be the wrong reaction. So far, the discussion has assumed that the right assignment of object to a particular token episode of demonstrative thought is the assignment that maximises the knowledge possessed by the thinker during that very episode. But charity-based or interpretationist approaches to content determination are typically more holistic. The unit of interpretation is typically taken to be something larger than a single episode of thought.

24 McGlynn 2012 objects to Williamson's knowledge maximisation theory with examples somewhat like the one just given, in which it seems that demonstrative reference persists although knowledge is undercut. 
If so, then when it comes to context-sensitive types of thought, like perceptual demonstrative thought, we are not forced to hope that for each episode of thought, we can find an object of thought that maximises the thinker's knowledge during that episode. Rather we can look at a much wider range of episodes, in the life of the individual creature, or community, and assign to the thought type a context-independent rule or character, which determines for each episode of that type the object of the episode of thought, in a way that maximises knowledge overall across the whole range of such episodes.

I believe that this is one way (perhaps not the only way) that a theory of thought determination can engage with the point that we human beings are commonly much more interested in material constitutees than hunks of matter. Although cases of sortal ignorance can happen, our perceptual demonstrative episodes are in a massively predominant range of cases responding to channels of knowledge of sort-proprietary properties of material constitutees, and not the coincident hunks of matter: our interests lead us to think a multitude of thoughts like 'that is alive', 'that is pretty', that isn't working properly', thoughts that $d_{0}$ count as knowledge, but only on assignment of the material constitutee to 'that'. So, the epistemic best candidate rule of reference for that type of thought overall is plausibly the following: the object of an episode of perceptual demonstrative thought is the material constitutee perceptually selected. This is the assignment of reference rule to the perceptual demonstrative type of thought that, given our practical interests, maximises knowledge across the whole range of episodes of that type. ${ }^{25}$

So, in the case of demonstrative thought about one of two coincident material things in ignorance of its sort, knowledge maximisation can indirectly make the statue the object of thought even when the subject doesn't in that situation know anything about the statue that discriminates it from the hunk of clay. The subject only knows how it looks and where it is. The close proximity of immaterial dead ringers could undermine any other knowledge which on that occasion might promise to discriminate it from the hunk of clay. The statue is nevertheless

25 A slightly less natural but even more epistemically charitable rule would be this: the object of an episode of perceptual demonstrative thought is the material constitutee perceptually selected, or, if no material constitutee is perceptually selected, whatever else is perceptually selected. This assignment tends to make episodes of demonstrative thought attempted on the basis of awareness of optical objects like holograms knowledgeable too. 
rightly counted as the object of thought: it is the object determined by the rule that maximises knowledge overall across all episodes of that type of thought. The statue is the material constitutee perceived.

We have here a theoretical vindication of Evans's suggestion that statues or living organisms somehow outrank by default the associated hunks of matter in cases of demonstrative identification in sortal ignorance. Could this ranking be overturned? There are particular occasions on which a butcher or a scrap-metal merchant might be more interested in the hunk of matter than in the material constitutee. But given the predominance of human interest in material constitutees, the overall charitable reference rule for the perceptual demonstrative type remains focused on material constitutees, so the thinker on these occasions would need to employ some further act of conceptual selection to express his interests: 'the hunk of flesh here', 'the piece of metal making up this [a chair, say]'. It is hard to imagine a kind of organism that would not predominantly take an interest in material constitutees (in its conspecifics, predators and prey) but one can just about envisage a kind of totally solitary creature interested only in gathering hunks of edible matter of any form. Perhaps the default objects of perceptual demonstrative thought for such creatures would be hunks of matter. But we are not like that.

\section{Conclusion}

I have sketched a solution to the selection problem for pluralism. It does not insist on correct sortal classification at any level of generality, nor does it claim that our perceptual systems alone suffice to select between coincident material things. It appeals to a general epistemic approach to the determination of thought content to explain why the statue is the default object of thought.

Note that, according to this solution, the thinker need not grasp the category material constitutee, despite its theoretical significance. The category is mentioned in stating the rule, but the thinker (as opposed to the theorist of content determination) need not grasp that this is the rule governing her thought. The factors that determine this to be the rule are factors largely 'outside the head' of the thinker on a given occasion of perceptual demonstrative thought: factors concerning the overall knowledge maximisation of a much wider range of episodes. 
With this noted, we can return to the way in which pluralism conflicts with pre-theoretical appearances, mentioned at the end of the section on perceptualism. When faced with a statue in normal conditions, it seems as if vision alone enables one to select a single object of thought; it does not seem as if anything more is needed for one's 'this'thought to lock onto a particular material thing. We can now see more clearly why things seem that way. First, the problem to be solved is undetectable to pre-theoretical reflection: the duality of candidate objects of thought is visually undetectable. Second, we can now see why the means by which the problem is solved is also undetectable to pre-theoretical reflection: the further selection is achieved not by any introspectable act of classification on the part of the thinker but by highly externalistic factors that are inscrutable from one's here-andnow perspective. That's why it seems as if vision alone enables singling out.

It is conceded, then, that pluralism is counterintuitive, in a way that goes well beyond the inevitable but superficial strangeness of the idea of two material things sharing their matter and location; pluralism conflicts with the pre-theoretical appearance that presence to the senses alone enables thought about the ordinary material things of human interest. What we have now seen is that a principled pluralist explanation of the possibility of selective thought about coincident material things can explain this pre-theoretical appearance. 